\title{
Response analysis of random base excitation ona magnetic bearing rotor system
}

\author{
Jinping Chen ${ }^{a}$, Li Zhang ${ }^{b}$, Yanyan Luo ${ }^{c}$,Haining Zhang ${ }^{d}$, Jun Liu ${ }^{e}$ \\ AVIC Qing 'an Group Co., Ltd., 710077, Xi'an, China. \\ achenjp88@163.com, ${ }^{b} 19871027 z h a n g l @ @ 163 . c o m,{ }^{c} 920510397 @ q q . c o m,{ }^{d}$ a335342n@126.com, ${ }^{\text {e } 175230504 @ q q . c o m, ~}$
}

\begin{abstract}
The magnetic bearing-rotor system is subject to various external disturbances in practical application. Under certain control conditions, the random response characteristics of the magnetic bearing-rotor system are a particular concern. This paper analyzes the response characteristics of base of the magnetic bearing subjected to acceleration random excitation in the horizontal direction. First, the magnetic bearing-rotor system model is deduced. Then, the random response of the rotor under acceleration random excitation is derived. The probability of the collision of the rotor between the auxiliary bearing is calculated and the example is given. The paper conclusion provides a theoretical basis for the collision detection and prediction of the magnetic bearing-rotor system.
\end{abstract}

\section{Introduction}

Magnetic Bearing uses the magnetic force to suspend the rotor in the air, so that there is no mechanical contact between the rotor and the stator. Compared with the traditional ball bearing, sliding bearing and oil film bearing, the magnetic bearing has no mechanical contact. The rotor can run to a high speed. It has the advantages of small mechanical wear, low energy consumption, low noise, long life, no lubrication, no oil pollution and so on. So it is especially suitable for high speed, vacuum and ultra clean environment. The theoretical research and Application Research of magnetic suspension bearings have been continued until now. Because most of the magnetic levitation bearings are used in high-speed rotating machinery, the working gap of the magnetic bearing is very small. The high speed rotor produces violent vibration during the fall process, which can cause damage to the rotor and auxiliary bearings, even result in the system failure. Therefore, it is very important to predict the probability of the collision between the rotor and the auxiliary bearing in the practical application of the magnetic bearing. There are only a few works in the literature in this respect. This paper tries to make a preliminary study on this aspect and analyzes the probability of collision between the rotor and the auxiliary bearing under base of the magnetic bearing subjected to the random excitation conditions.

\section{Axial magnetic bearing model}

As shown in FIG. 1, the magnetic bearing system consists of a rotor, a thrust disk, an axial magnetic bearing, an auxiliary bearing, a sensor, an amplifier, and a controller.

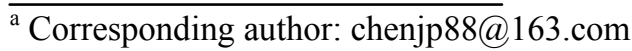

The mathematical model of the axial magnetic suspension bearing is[1,2]

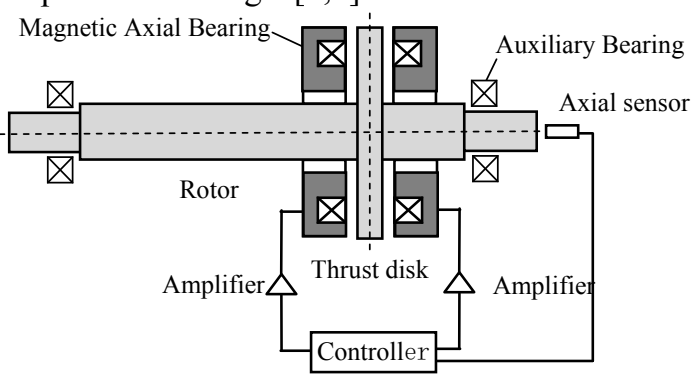

Fig 1Schematic diagram of magnetic bearing rotor system Usually, two counteracting magnets are operated in a bearing magnet (see the geometry in Fig.2). This configuration makes it possible to generate both positive and negative forces. In the case of the so-called differential driving mode, one magnet is driven with the sum of bias current $i_{0}$ and control current $i_{x}$, and the other one with the difference $\left(i_{0}-i_{x}\right)$. Consequently, if the magnetization of the iron is neglected, this scheme produces a linear force-current relation as discussed below.

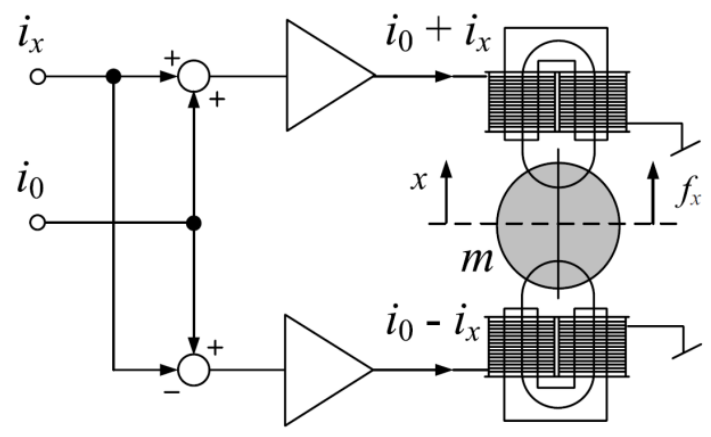

Fig 2 Differential driving mode of the bearing magnets 
Force $f_{x}$ in Fig. 3 represents the difference of forces between both magnets. Both forces are obtained by the sum $\left(i_{0}+i_{x}\right)$ and the difference $\left(i_{0}-i_{x}\right)$. For the air gaps, $\left(x_{0}\right.$ $+x)$ and $\left(x_{0}-x\right)$ :

$f_{x}=f_{+}-f_{-}=\frac{\mu_{0} n^{2} A}{4}\left[\frac{\left(i_{0}+i_{x}\right)^{2}}{\left(x_{0}+x\right)^{2}}-\frac{\left(i_{0}-i_{x}\right)^{2}}{\left(x_{0}-x\right)^{2}}\right]$

where: $\mu_{0}$ is the vacuum permeability, $n$ is the number of coil turns, $A$ is the air-gap cross-sectional area.

Equation (1) is a non-linear equation. Because the working gap of the magnetic bearing is very small, it can be liberalized at the work center position, and using the Taylor expansion, the force equation in Eq. (1) can be linearized a for small perturbation current $i_{0}$ around the bias current and the nominal air gap $x=0$. By expanding the force equation in Eq. (1) in terms of the Taylor series around the point $\left(i_{0}, x_{0}\right)=(0,0)$, and ignoring the second and higher order terms, we obtain the following expression for the force:

$$
f_{x} \approx k_{x} x+k_{i} i
$$

where $k_{x}=-\frac{\mu_{0} n^{2} A i_{0}^{2}}{x_{0}^{3}}$ isthe force-displacement stiffness coefficient. $\quad k_{i}=\frac{\mu_{0} n^{2} A i_{0}}{x_{0}^{2}} \quad$ is force-current stiffness.

When studying the magnetic bearing system, it is often compared with similar mechanical systems. Because mechanical system model has a very complete maturity theory. A typical single-degree-of-freedom mechanical system consists of three basic elements: the mass $m$, spring $k$, damping $c$. The mechanical system motion differential equation is[3]

$$
m \ddot{x}+c \dot{x}+k x=f
$$

For an axial magnetic bearing rotor system, its differential equation of motion is

$$
m \ddot{x}+k_{x} x+k_{i} i_{x}=f
$$

Since the rotor system supported by the axial magnetic suspension bearing can be regarded as a single-degree-of-freedom mechanical system, the two systems are equivalent.

then

$$
c \dot{x}+k x=k_{x} x+k_{i} i_{x}
$$

So the control current should follow:

$$
i_{x}=\frac{\left(k-k_{x}\right) x+c \dot{x}}{k_{i}}=P x+D \dot{x}
$$

The formula (6) shows that the control current $i_{x}$ needs at least proportional control and differential control.

Obviously, once the control parameters $P$ and $D$ are given, $k$ and $c$ in the mechanical model equivalent to the typical single degree of freedom can be determined.The response characteristics of the equivalent single-degree-of-freedom mechanical system model under random excitation disturbance conditions are studied below.

\section{SDOF with random enforced acceleration model[4]}

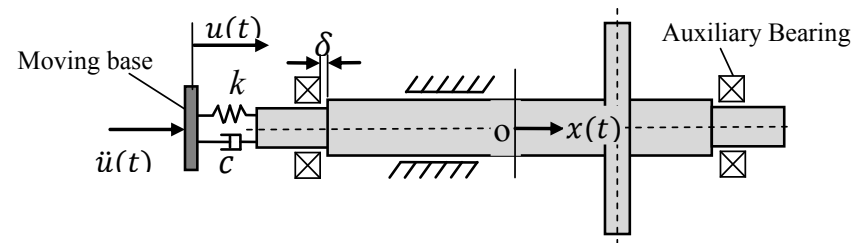

Fig 3 the single-degree-of-freedom mechanical system model

This paper discuss the probability of the collision between the rotor and the auxiliary bearing when the foundation is subjected to random excitation. For this reason, the schematic diagram of Figure 1 is changed to Figure 3, in which the basic part model is added.

The system model can be considered as a single-degree-of-freedom system, a SDOF system with a discrete mass $m$, damper element $c$ and spring element $k$ is placed on a moving base with an acceleration $\ddot{u}(t)$. The resulting displacement of the mass is $x(t)$. A relative motion $z(t)$ will be introduced which is the displacement of the mass with respect to the base. The relative displacement is

$$
\mathrm{z}(\mathrm{t})=\mathrm{x}(\mathrm{t})-\mathrm{u}(\mathrm{t})
$$

The equation of motion of the SDOF system, illustrated in Fig.2, is

$$
\mathrm{m} \ddot{\mathrm{x}}(\mathrm{t})+\mathrm{c}\{\dot{\mathrm{x}}(\mathrm{t})-\dot{\mathrm{u}}(\mathrm{t})\}+\mathrm{k}\{(\mathrm{x}(\mathrm{t})-\mathrm{u}(\mathrm{t})\}=0
$$

Using (7) we can write the equation of motion (8) of the SDOF system as

$$
\ddot{\mathrm{z}}(\mathrm{t})+2 \xi \omega_{\mathrm{n}} \dot{\mathrm{z}}(\mathrm{t})+\omega_{\mathrm{n}}^{2} \mathrm{z}(\mathrm{t})=-\ddot{\mathrm{u}}(\mathrm{t})
$$

The enforced acceleration of the SDOF system is transformed into an external force.The Fourier transform of (9) will lead to the following equation of motion in the frequency domain

$$
\left[-\omega^{2}+2 j \xi \omega \omega_{n}+\omega_{n}^{2}\right] Z(\omega)=-\ddot{U}(\omega)
$$

or

$$
Z(\omega)=\frac{-\ddot{U}(\omega)}{-\omega^{2}+2 j \xi \omega \omega_{n}+\omega_{n}^{2}}=-H(\omega) \ddot{U}(\omega)
$$

\section{Statistical solution of random responses [5]}

The PSD function of $z(t)$ is given by 
$S_{\mathrm{ZZ}}(\omega)=\lim _{\mathrm{T} \rightarrow \infty} \frac{1}{\mathrm{~T}} \mathrm{Z}(\omega) \mathrm{Z}^{*}(\omega)$

and the PSD function of $\ddot{u}(t)$ is given by the following equation

$S_{\ddot{u} \ddot{u}}(\omega)=\lim _{T \rightarrow \infty} \frac{1}{T} \ddot{U}(\omega) \ddot{U}^{*}(\omega)$

We can write the PSD function $S_{z z}(\omega)$ of the relativemotion $z(t)$ as

$$
S_{\mathrm{zz}}(\omega)=|H(\omega)|^{2} S_{\ddot{u ̈ u ̈}}(\omega)
$$

We shall derive of the mean square values both in the time and frequency domain starting from the autocorrelation function $R_{z z}(\tau)$

$R_{z Z}(\tau)=\frac{1}{2 \pi} \int_{-\infty}^{\infty} S_{z z}(\omega) e^{j \omega \tau} d \omega$

and inserting (14) we find the following expression:

$$
R_{z z}(\tau)=\frac{1}{2 \pi} \int_{-\infty}^{\infty}|H(\omega)|^{2} S_{\ddot{u} \ddot{u}}(\omega) e^{j \omega \tau} d \omega
$$

or with a constant PSD function $S_{\ddot{u} \ddot{u}}(\omega)=$ $S_{\ddot{u}}=\frac{w_{\ddot{u}}}{2}$

$R_{z z}(\tau)=\frac{s_{\ddot{u}}}{2 \pi} \int_{-\infty}^{\infty}|H(\omega)|^{2} e^{j \omega \tau} d \omega$

Equation (11)gives

$$
\begin{aligned}
& |H(\omega)|^{2}=\frac{1}{\left(\omega_{n}^{2}-\omega^{2}\right)^{2}+\left(2 \xi \omega \omega_{n}\right)^{2}}= \\
& \frac{1}{\omega_{n}^{4}\left[\left(1-\frac{\omega^{2}}{\omega_{n}^{2}}\right)^{2}+\left(2 \zeta \frac{\omega}{\omega_{n}}\right)\right]}
\end{aligned}
$$

Finally the autocorrelation function $R_{z z}(\tau)$ is obtained

$$
\begin{gathered}
R_{z z}(\tau)=\frac{s_{\ddot{u}} e^{-\xi \omega_{n} t}}{4 \zeta \omega_{n}^{3}}\left\{\cos \left(\omega_{n} \sqrt{1-\xi^{2}}\right) t\right\}+ \\
\frac{\xi}{\sqrt{1-\xi^{2}}} \sin \left(\omega_{n} \sqrt{1-\xi^{2}}\right) t
\end{gathered}
$$

The mean square response of the relative displacement $z(t)$ is

$$
E\left\{z(t)^{2}\right\}=R_{z z}(0)=\frac{s_{\ddot{u}}}{4 \xi \omega_{n}^{3}}=\frac{W_{\ddot{u}}}{8 \xi\left(2 \pi f_{n}\right)^{3}}
$$

The derivation of the mean square calculation of $z(t)$ in the frequency domain is repeated. With reference to (16) the mean square response of $z(t)$, due to the random excitation $\ddot{u}(t)$,with the PSD function $S_{\ddot{u} \ddot{u}}(\omega)$, becomes

$E\left\{z(t)^{2}\right\}=R_{z z}(0)==$

$\frac{1}{2 \pi} \int_{-\infty}^{\infty}|H(\omega)|^{2} S_{\ddot{u} \ddot{u}}(\omega) d \omega$

If the enforced acceleration $\ddot{u}(t)$ has a constant PSD function (white noise) $S_{\ddot{u} \ddot{u}}(\omega)=S_{\ddot{u}}=\frac{w_{\ddot{u}}}{2}$, then

$E\left\{z(t)^{2}\right\}=R_{z z}(0)=\frac{s_{\ddot{u}}}{2 \pi} \int_{-\infty}^{\infty}|H(\omega)|^{2} d \omega$

This integral (22) has a known solution

$E\left\{z(t)^{2}\right\}=R_{z z}(0)=$

$\frac{s_{\ddot{u}}}{2 \pi} \int_{-\infty}^{\infty}|H(\omega)|^{2} d \omega=\frac{S_{\ddot{u}}}{4 \xi \omega_{n}^{3}}=\frac{W_{\ddot{u}}}{8 \xi\left(2 \pi f_{n}\right)^{3}}$

\section{Andvariance:}

$$
\sigma^{2}=E\left\{z(t)^{2}\right\}-E^{2}\{z(t)\}
$$

In general since the excitation is a Gaussian process, the probability density function of the response also obeys a Gaussian distribution.

$P_{\mathrm{z}}=\frac{1}{\sqrt{2 \pi} \sigma} \exp \left[-(z(t)-E[z(t)])^{2} / 2 \sigma^{2}\right]$

Therefore, the probability that the random response is greater than the gap $\delta$ is

$P(|z(t)|>\delta)=1-\frac{1}{\sqrt{2 \pi} \sigma} \int_{-\delta}^{\delta} \exp [-(z(t)-$
$\left.E[z(t)])^{2} / 2 \sigma^{2}\right] d z$

\section{Example}

Suppose the system in Fig. 2 has a natural frequency $f_{n}=350 \mathrm{~Hz}$ and a damping ratio $\xi=0.025$ the clearance between the rotor and the auxiliary bearing is $\delta=$ $0.2 \mathrm{~mm}$.when the base excitation is a stationary random acceleration with a mean of zero PSD spectral density as show table 1or Fig 4, What is the probability that the system will not collide?

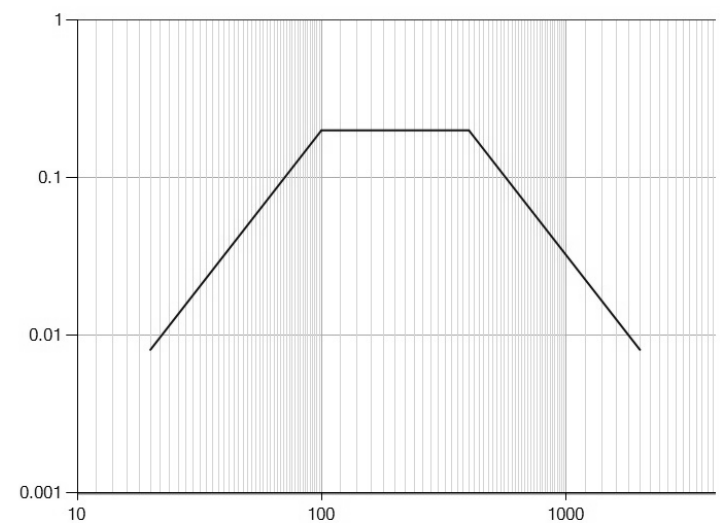

Fig 4 PSD spectrum acceleration specification 
The power spectral density of random vibration, which is very common in engineering, is shown in Fig. 4. In practical use, it is converted into specific values. The converted values in this example are shown in Table 1.

Table 1 PSD spectrum acceleration

\begin{tabular}{|l|l|}
\hline Frequency $(\mathrm{Hz})$ & $\begin{array}{l}\mathrm{PSD} \mathrm{g} / \mathrm{Hz} \text { (single } \\
\text { sided) }\end{array}$ \\
\hline $20-100$ & $6 \mathrm{~dB} /$ oct \\
\hline $100-400$ & 0.2 \\
\hline $400-2000$ & $-6 \mathrm{~dB} /$ oct \\
\hline
\end{tabular}

Mean squared error is

$$
\begin{gathered}
E\left\{z(t)^{2}\right\}=\frac{W_{\ddot{u}}}{8 \xi\left(2 \pi f_{n}\right)^{n}}=\frac{0.20 \times 9.81^{2}}{8 \times 0.025 \times(2 \pi 350)^{3}} \\
=9.06 \times 10^{-9} \mathrm{~m}^{2}
\end{gathered}
$$

The standard deviation of the displacement is

$\sigma=\sqrt{E\left\{z(t)^{2}\right\}}=\sqrt{9.06 \times 10^{-9}}=9.52 * 10^{-5} \mathrm{~m}$

$y=\frac{\delta-0}{\sigma}=\frac{0.0002-0}{9.52 * 10^{-5}}=2.101$

the probability that Prob:

$$
\begin{aligned}
\{|\mathrm{z}(\mathrm{t})|>0.0002\} & =P(|\mathrm{z}|>0.0002) \\
= & 1-\frac{1}{\sqrt{2 \pi}} \int_{-2.101}^{2.101} e^{-y^{2} / 2} d y \\
= & 0.0357
\end{aligned}
$$

In the example, the probability of collision between the rotor and the auxiliary bearing is not large, It shows that the design of the magnetic bearing system in this example is basically reasonable, and it can operate safely in actual use and it can be further based on $3 \sigma$ or $6 \sigma$ rules to determine the protection gap between the rotor and the auxiliary bearing.

\section{Conclusion}

This paper analyzes the random response characteristics of the axial magnetic levitation system under base with an random acceleration excitation and gives the control model of the basic magnetic levitation system. According to the equivalent principle, the corresponding one-degree-of-freedom mechanical system mechanical model is determined, the response characteristics under base with an random acceleration excitation of model is analyzed. The probability formula is given for predicting the collision between the rotor and the auxiliary bearing. At the same time, a calculation example is given.

It can also be based on $3 \sigma$ or $6 \sigma$ rules combines the requirements of the levitation force to consider the design of the clearance between the rotor and the auxiliary bearing, the paper provide a new attempt for the design of the magnetic suspension bearing rotor system.

\section{References}

1. G. H. Schweitzer, E. H. Maslen, "Magnetic Bearings: Theory, Design andApplication to Rotating Machinery", 2009, Springer-Verilog, Berlin, Heidelberg

2. JinpingChen,"Random response analysis of magnetic bearing rotor system" the 16th International Symposium on Magnetic Bearings (ISMB16), Beijing, China, August 13-17, 2018.

3 .S.Timoshenko:"Vibrationproblem in Engineering",1937

4. Crandall SH, Mark WD "Random vibration in mechanical systems". 1973 Academic Press

5. JaapWijker. "Random Vibrations in Spacecraft Structures Design". SpringerScience + Business Media 